\title{
Novel and Simple Patterning process of Quantum Dots via Transfer Printing for Active Matrix QD-LED
}

\author{
Sang Yun Bang, Xiangbing Fan, Sang Hyo Lee, Hyung Woo Choi, Dong Wook Shin, \\ Sung Min Jung, Hanleem Lee, Jiajie Yang, Yo-han Suh, Luigi G. Occhipinti, \\ Soo Deok Han*, and Jong Min Kim \\ *Electrical Engineering Div., University of Cambridge, Cambridge, UK
}

\begin{abstract}
The next generation of a self-emitting display requires precise and stable patterning techniques to shape Red, Green, and Blue pixels using quantum dots. In this study, we propose the novel and simple transfer printing process for the active matrix $Q D$ LEDs.
\end{abstract}

\section{Author Keywords}

Quantum dots; Quantum dots light emitting diode; Transfer printing; Patterning techniques; Electroluminescence; Displays

\section{Introduction}

Colloidal quantum dots (QDs) are prosperous material for the next generation electroluminescent display application with the advantages such as a colour tunability, high luminescence and sharp emission bandwidth. ${ }^{1}$ Since the QDs, which is the most important emission layer of QD-LED, is limited to make as the solution by synthesis process, so far, the process of making QDLED has been mainly developed by the solution process such as the spin coating. ${ }^{2}$ Even though the spin coating has been attractive due to the easy control of composition and low-cost, nevertheless, there is a limit to ensuring the stability of the QDLED, which consider the suitability of the solvent of the subsequent layer to the lower layer and require multiple processes with chemicals. Moreover, it is very challenging to apply photolithography as a conventional patterning process which critically affects the stability of QDs.

For the QDs based next generation display, a precise and stable patterning method with the red, green, and blue QDs should be developed. So far, the inkjet printing, direct photolithography mixed with photosensitive resin, and transfer printing have been developed to pattern quantum dots. ${ }^{3-6}$ Among them, the transfer printing is a novel patterning process which transfers the precoated QDs layer on a donor substrate to the desired place. ${ }^{6}$ Furthermore, the dry process fosters lithography free QDs film processing to respond room temperature, close-packed density and void free QDs layer, which will enable numerous form factor from solid substrate to the flexible device while maintaining the advance quality of QDs layers. ${ }^{5}$

The transfer printing has been developed as an embossment (to use a patterned stamp and transfer QDs layer from the donor substrate to the desired substrate) and intaglio (to firstly pick-up and drop-off the QDs on the donor substrate via a reverse shaped stamp, and secondly transfer the residual QDs layer via the flat stamp to the desired substrate).$^{6-7}$ However, a big issue of transfer printing is that much QDs are discarded after pick-up and it has not been considered to utilize residual QDs of the donor substrate. For example, to fabricate an active matrix display consisting of RGB with an aperture ratio of $50 \%$, if the one colour of QDs is transferred from the donor substrate, then the remaining $83.33 \%$ of the QDs should be discarded. State of the art in the transfer printing process has been focused only on patterning and not considered much of waste. In this study, we firstly developed a novel and simple transfer printing technique that minimizes QDs loss, which includes both embossment and intaglio methods. Moreover, we demonstrated that a platform where the desired shape can be precisely placed on the active matrix display could be easily fabricated by rotating a single striped-PDMS stamp only 90 degrees in the direction.

\section{Results and Discussion}

The transfer printing process consists of the preparation of donor substrate, PDMS stamp, ITO substrate deposited the HIL (PEDOT:PSS) and HTL (TFB) layer, transfer printing, and subsequent deposition of the ETL ( $\mathrm{Al}$ doped $\mathrm{ZnO}$ ) and $\mathrm{Al}$ electrode for the bottom emission QD-LED. The overall structure of QD-LED is shown in Figure 1. In this study, we have mainly tried to make QD-LED by transferring QD pixel via patterned PDMS stamp via the transfer printer (Seoul Engineering Co LTD, Figure 2), and apart from that we also have approached the new direction for patterned QDs pixel.
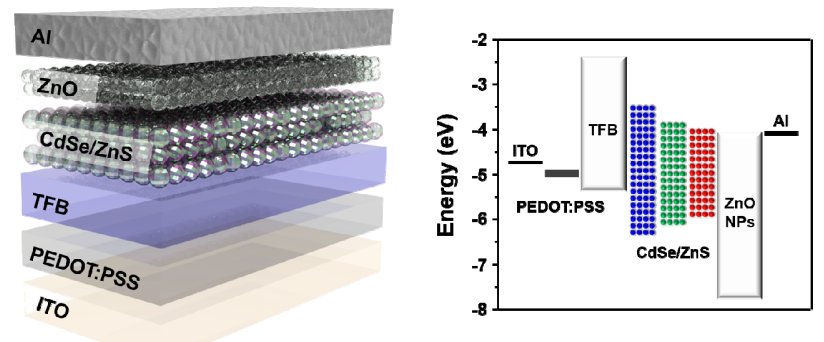

Figure 1. Overall QD-LED structure via the transfer printer

Utilizing the transfer printing technique, the passive QLED was fabricated to characterize the performance of a unit device itself. As following a conventional recipe for PM-QLED, the patterned the ITO substrates $(100 \mathrm{~nm})$, were spin-coated with PEDOT: PSS (2000 rpm for $45 \mathrm{sec}, 150^{\circ} \mathrm{C}$ for $30 \mathrm{~min}$ ) for HTL, and TFB (2000 rpm for $45 \mathrm{sec}, 130^{\circ} \mathrm{C}$ for $30 \mathrm{~min}$ ) for HIL. The donor substrate was prepared to have hydrophobic surface by ODTS wet-solution method, which activates the oriented surface coating using non-polar solvents. A self-assembly monolayer (SAM) was formed on the silicon substrate surface using the Octadecyltrichlorosilane (ODTS) to make the donor substrate. Then, the QDs layer was spin-coated on the Si substrate, which a SAM of ODTS was grown beforehand. The SAM acts as a buffer layer between the QDs and silicon and helps in the peeling off the QDs by reducing the surface energy on silicon. The CdSe/ZnS based red QD, the concentration of $12.5 \mathrm{mg} / \mathrm{ml}$, 
was spin-coated for the final step. After picking and transferring the patterned QDs (Pressure - Pick-up $0.5 \mathrm{~kg}$ for $5 \mathrm{sec}$, Transfer $2.0 \mathrm{~kg}$ for $30 \mathrm{sec}$, Peeling velocity: Pick-up $500 \mathrm{~mm} / \mathrm{s}$, Transfer $1 \mathrm{~mm} / \mathrm{s}$ ), the $\mathrm{Al}$ doped $\mathrm{ZnO}$ nanoparticles was spin-coated for ETL layer (2000 rpm for $45 \mathrm{sec}, 80^{\circ} \mathrm{C}$ for $15 \mathrm{~min}$ ), finally the $\mathrm{Al}$ electrode $(200 \mathrm{~nm})$ was deposited by e-beam evaporator under $1.0 \times 10^{-6}$ torr.

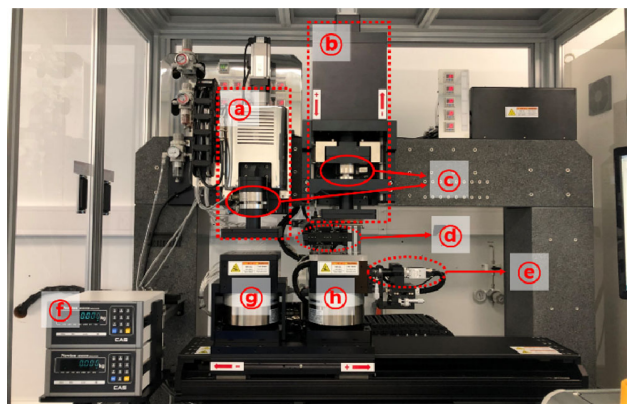

(a) High speed module with solenoid pump for the pick-up (Z1 axis)

(b) Low speed module with linear motor for the transfer (22 axis)

(c) Loadcell - Pressure sensor

(d) Upper optics - Two CCD cameras for the top and bottom view

(e) Lower optics - CCD camera

$($ Pressure indicator

(a) Substrate holder for the pick-up ( $\mathrm{X}$ and $\mathrm{Y}$ axis)

(D) Substrate holder for the alignment and transfer ( $\mathrm{X}$ and $\mathrm{Y}$ axis)

Figure 2. Overview image and description of the transfer printer

The electroluminescence performance was shown as a lowvoltage turn-on at $2.2 \mathrm{~V}$ with the maximum EQE of $4.33 \%$ (Figure 3). The peak wavelength was measured at $651 \mathrm{~nm}$ with FWHM $31.5 \mathrm{~nm}$ at $600 \mathrm{~cd} / \mathrm{m} 2$, which fitted under the range of red wavelength. A High density ODTS self-assembly monolayer treatment enhanced not only the surface uniformity of dry transfer printing process, but also the closed packing density of QD molecules in a layer. For the further studies, the ODTS coating protocol can be prepared under either in nitrogen or in argon environment, which protect from the oxygen and water concentration for the best surface modification and reproducibility of superior device performance. These controlled experiments will give outstanding samples, which are competitive to the samples from the spin-coating method.

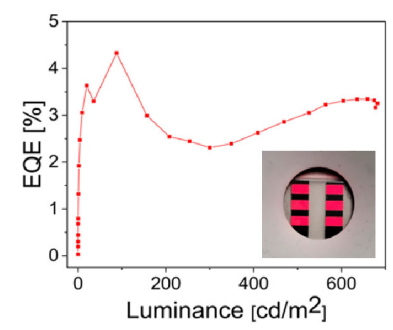

Figure 3. EQE of transfer printed red QD-LED as a function of luminance.
In order to develop a novel and efficient transfer printing process, we designed a simple striped pattern with a width of $200 \mu \mathrm{m}$ and a spacing of $200 \mu \mathrm{m}$ (Figure 4). Firstly, a $9 \mu \mathrm{m}$ height of striped PDMS stamp (emission area more than 1 inch) was prepared using SU8-2010 by the photolithography process. Using one stamp, we have studied a new patterning process for picking-up and transferring QDs layer on a previously used donor substrate via a new striped PDMS stamp.

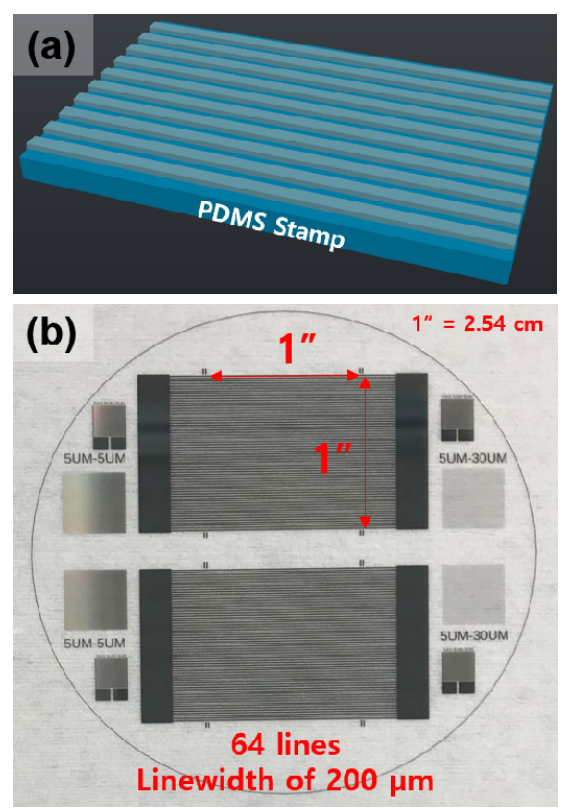

Figure 4. (a) Schematic of PDMS stamp. (b) Image of photo mask for the $200 \mu \mathrm{m}$ striped pattern.

Figure 5 is the schematics of the whole patterned printing process. First, the first QD layer from the donor substrate was picked up using the striped PDMS stamp mentioned above. As a result, a striped pattern with a spacing of $200 \mu \mathrm{m}$ remains on the donor substrate, as shown in Figure 5 (a). Next, using the second new striped PDMS stamp, we rotated at $90^{\circ}$ in the first pick-up direction and made a second pick-up. As a result, the striped PDMS stamp was picked up only in the remaining $200 \times 200 \mu \mathrm{m}$ QD layer, and the same QD pattern remained on the donor substrate. In addition, using a new flat PDMS stamp, the third process could be used to pick-up and transfer the remaining 200 x $200 \mu \mathrm{m}$ pattern on the donor substrate. The inset Figures 5 (a) and (b) show the optical PL images of donor substrate and PDMS stamp for each step.

The advantage of this method is that it can be obtained three kinds of QD patterned PDMS stamp in simple ways, and it is a cost-effective way to use QD remaining on the donor substrate without waste. PM-QLED was fabricated by aligning and transferring the square-shaped $(200 \times 200 \mu \mathrm{m})$ QDs patterned PDMS. Figure 6 (a) shows the PL image after picking-up the RGB QDs on the PDMS stamp, and Figure 10 (b) - (d) shows the optical images of the EL emission of pixelated QD-LED. 
(a) Donor substrate

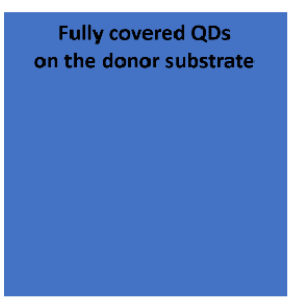

(b) PDMS stamp

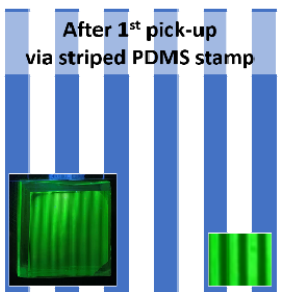

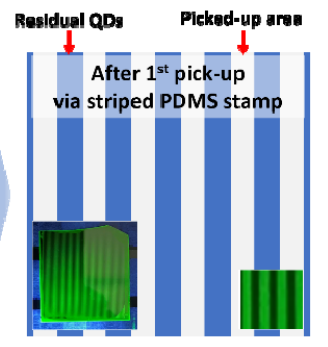

After $2^{\text {nd }}$ pick-up via striped PDMS stamp with rotated $90^{\circ}$
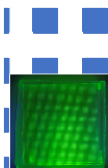

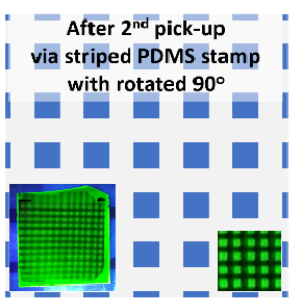

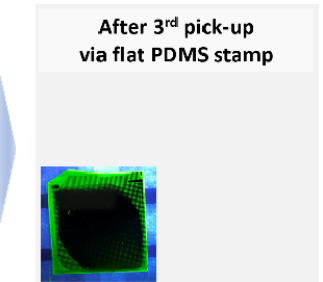

(c)

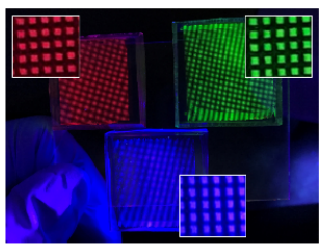

Figure 5. Overview of the transfer printing process via the crossing stripe patterned PDMS. (a) Schematics of the crossing stripes process on the donor substrate. Inset, the optical images of PL emission of the donor substrate. (b) Schematics of the crossing stripes process on the PDMS stamp. Inset, the optical images of PL emission of PDMS stamp. (c) Optical image of the PL emission of RGB patterned PDMS stamp $(200 \times 200 \mu \mathrm{m})$.
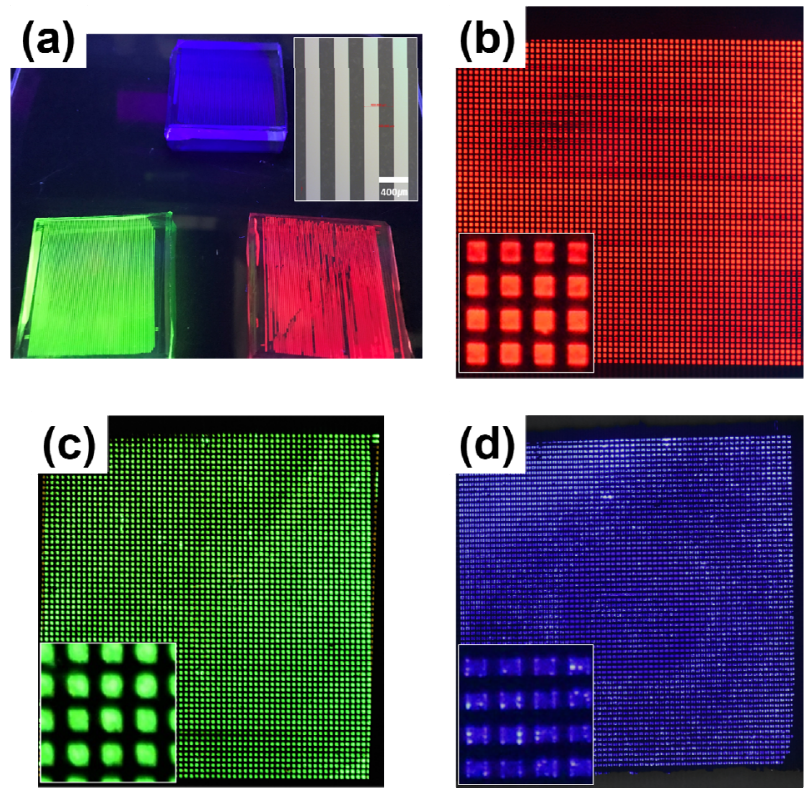

Figure 6. (a) Optical image of PL emission of the $200 \mu \mathrm{m}$ striped PDMS stamp (1 inch) after picking-up from the donor substrate. Inset, the microscope image of photomask for ITO and PDMS stamp pattern. (b) - (d) Optical images of the EL emission of Red, Green, and Blue on the 1 inch of PM-QLED after transferring.

Based on this result, it is possible to make R, G, and B patterned QDs pixels suitable for Active matrix QD-LED by differently adjusting the gap between the stripes rather than a striped pattern having the same width and spacing. If the stripe-patterned PDMS is picked up in the same way by designing strip patterns with a width of $100 \mu \mathrm{m}$ and gap of $300 \mu \mathrm{m}$, a pixelated QDs pattern of $100 \times 300 \mu \mathrm{m}$ can be obtained at the second pickingup. When picking up and transferring $\mathrm{R}, \mathrm{G}$ and $\mathrm{B}$ with the gap of $33.33 \mu \mathrm{m}$, we can make R, G, B patterned QLED with the same interval as the last image. Considering the space between QDs, it will be desirable to design TFTs and capacitors for active matrix for the bottom emissive QD-LED in the future.

\section{Conclusion}

In this study, beyond the conventional advantage of transferring QD to the desired area, we demonstrated that the layer of QDs can be efficiently transfer via one stamp without discarding the QDs, considering the design of active matrix QD-LED. This easy and simple patterning process into desired shapes has paved the new way for the development of the next generation of QD based displays.

\section{Acknowledgements}

The authors acknowledge the support from European Commission Horizon2020 under grant agreement number (685758). The research leading to these results received funding from Engineering and Physical Sciences Research Council (EPSRC) project reference of EP/P027628/1 for funding the 'Smart Flexible Quantum Dot Lighting' used in this research.

\section{Reference}

1. K.-S. Cho, E. K. Lee, W.-J. Joo, E. Jang, T.-H. Kim, S. J. Lee, S.-J. Kwon, J. Y. Han, B.-K. Kim, B. L. Choi, and J. M. Kim, "High-performance crosslinked colloidal quantum-dot light-emitting diodes", Nature Photonics, 3, 341-345 (2009). 
2. H. Shen, Q. Gao, Y. Zhang, Y. Lin, Q. Lin, Z. Li, L. Chen, Z. Zeng, X, Li, Y, Jia, S, Wang, Z. Du, L. S. Li, and Z.

Zhang, "Visible quantum dot light-emitting diodes with simultaneous high brightness and efficiency", Nature Photonics, 13, 192-197 (2019).

3. Y. Wang, I. Fedin, H. Zhang, and D. V. Talapin, "Direct optical lithography of functional inorganic nanomaterials", Science, 357, 385-388 (2017).

4. H. M. Haverinen, R. A. Myllylä, and G. E. Jabbou, "Inkjet Printed RGB Quantum Dot-Hybrid LED", JOURNAL OF DISPLAY TECHNOLOGY, 6, 3, 87-89 (2010).

5. T.-H. Kim, K.-S. Cho, E. K. Lee, S. J. Lee, J. Chae, J. W. Kim, D. H. Kim, J.-Y. Kwon, G. Amaratunga, S. Y. Lee, B. L. Choi, Y. Kuk, J. M. Kim, and K. Kim, "Full-colour quantum dot displays fabricated by transfer printing", Nature Photonics, 5, 176-182 (2011).

6. T.-H. Kim, D.-Y. Chung, J. Ku, I. Song, S.Sul, D.-H. Kim, K.-S. Cho, B. L. Choi, J. M. Kim, S. Hwang, and K. Kim, "Heterogeneous stacking of nanodot monolayers by dry pick-and-place transfer and its applications in quantum dot light-emitting diodes", Nature Communications, 4, 2637 (2013).

7. M. K. Choi, J. Yang, K. Kang, D. C. Kim, C. Choi, C. Park, S. J. Kim, S. I. Chae, T.-H. Kim, J. H. Kim, T. Hyeon and D.-H. Kim, "Wearable red-green-blue quantum dot light-emitting diode array using high-resolution intaglio transfer printing", Nature Communications, 6, 7149 (2015). 